\title{
6 Motivation und Einstellungen von Spanischlernenden: Methodisches zur Datenerhebung und -auswertung
}

Auch wenn das Spanische inzwischen in der schulischen Bildung einen festen Platz eingenommen hat, bleibt der tertiäre Bildungsbereich eine äußerst wichtige Umgebung für den Erwerb des Spanischen. Die Studienzeit ist beispielsweise für viele junge Menschen eine Phase, in der Sprachenlernen, die Verwendung von Fremdsprachen und die Begegnung mit der eigenen Mehrsprachigkeit besonders relevant werden (Vogl 2018a, b). Studierende sind deshalb eine interessante Zielgruppe zur Untersuchung von Spracheinstellungen im Lernprozess und zum Vergleich mit anderen Gruppen von Lernenden.

Hochschulen, Volkshochschulen oder private Sprachschulen sind die Orte, an denen Spanischkenntnisse aus der Schulzeit ausgebaut werden können, oder das Spanischlernen begonnen wird, wenn dies in der Schule nicht möglich war bzw. wenn Bedarf oder Interesse erst später aufkam. Auch in der Schweiz lässt sich nachweisen, dass Spanisch besonders häufig im tertiären Bildungswesen erlernt wird (Werlen 2010: 53). Auf diesen Bereich konzentrieren sich deshalb die Teilstudien, die in den folgenden Kapiteln vorgestellt werden.

Um Spracheinstellungen zum Spanischen sowie Lernziele und -motivationen von Lernenden zu untersuchen, wurden drei verschiedene Zielgruppen befragt:

1. Studierende der Freien Universität Berlin, die spanische Philologie oder Spanisch mit Lehramtsoption als Studienfachgewählt haben

2. Studierende verschiedener Fächer und Hochschulen, die an der Technischen Universität Berlin an der Sprach- und Kulturbörse einen Spanischkurs besuchen

3. Teilnehmer*innen von Spanischkursen an verschiedenen Volkshochschulen in Berlin

Bei der Befragung der verschiedenen Gruppen von Spanischlernenden an Hochschulen und Volkshochschulen wurde auf weitgehend gleich aufgebaute Fragebögen zurückgegriffen, die lediglich an den notwendigen Stellen der Zielgruppe angepasst waren. Der Aufbau der Bögen soll hier kurz erläutert und die darin enthaltenen Fragestellungen an die bestehende Forschung angebunden werden. Die Fragebögen sind im Anhang vollständig wiedergegeben.

Als identische Metadaten wurden von allen Befragten das Geburtsjahr und das Geschlecht erhoben. Alle Studierenden wurden gebeten, ihre Studienfächer anzugeben, die Lernenden an der VHS dagegen ihren höchsten erreichten 
Bildungsabschluss. Der allergrößte Teil der Fragebögen war durch geschlossene Fragen oder Aussagen mit vorgegebenen Items gestaltet. Damit wurden also Antwortmöglichkeiten zum Sprachenlernen und zur Sprachverwendung sowie zu Spracheinstellungen antizipiert und überprüft (vgl. Dörnyei/Taguchi 2010: 5-6). Insbesondere sicherte diese Vorgehensweise die Vergleichbarkeit der Daten zwischen den verschiedenen befragten Gruppen, die auf identische bzw. höchstens minimal an die Einzelgruppe angepasste Items reagierten (Adler/ Plewnia 2018: 70, 94). Vereinzelt wurde auf offene Fragen zurückgegriffen, um die Antwortmöglichkeiten nicht zu stark einzuschränken bzw. um eine möglichst breite Streuung von Antworten zu erhalten. Die Themenbereiche und die Einbindung der jeweils ausgewählten Items in den wissenschaftlichen Zusammenhang wird in den folgenden Abschnitten dargestellt.

Eine weitere Fragebogenstudie mit Beschäftigten im Berliner Gastgewerbe wird weiter unten vorgestellt. Der etwas andere Aufbau der Befragung und die unterschiedliche Methodik wird im entsprechenden Kapitel erläutert, so dass es hier vorerst nur um die Befragung von Spanischlernenden in unterschiedlichen Kontexten in Berlin gehen soll. Um die Fragestellungen im Einzelnen deutlich zu machen, ist die Erläuterung des Fragebogens in den folgenden Unterkapiteln als integrative methodisch-theoretische Darstellung jeweils verknüpft mit den zugrundeliegenden Konzepten und Begriffen.

Unter Motivation ist in Rahmen dieser Studie nicht in erster Linie der psychologisch bedingte Antrieb $\mathrm{zu}$ sehen, sich in die konkrete Lernsituation zu begeben, also mögliche Lernhindernisse zu überwinden und den Lernprozess $\mathrm{zu}$ beginnen oder fortzusetzen. Vielmehr erfasst Motivation hier die Gründe zum Erlernen, also diejenigen Faktoren, die dazu beitragen, das Erlernen einer Sprache überhaupt ins Auge zu fassen (vgl. zu diesem Themenbereich umfassend Dörnyei/Ushioda 2011).

In der Literatur wird häufig von zwei grundlegenden Faktoren ausgegangen, welche zur Motivation beim L2-Erwerb beitragen: Die integrativeness der Lernenden und ihre Einstellungen zum Lernprozess (Gardner 2001: 4-5). Der zweite Bereich soll im nachfolgenden Teilkapitel als erstes eingeführt werden, weil damit die Grundvoraussetzungen des Fremdspracherwerbs im Rahmen der jeweils gewählten Lernumgebung und Qualifikationsziele der verschiedenen Gruppen von Lernenden betrachtet werden. An zweiter Stelle steht die integrativeness, also die Absichten und Potenziale der Befragten, sich in die Sprachgemeinschaft der erlernten Sprache einzufügen und darin einen individuellen Platz einzunehmen (Dörnyei/Csizér 2002: 452-454). Hier spielen Einstellungen gegenüber der Sprache (und damit auch gegenüber der Sprachgemeinschaft) eine wichtige Rolle, der Faktor integrativeness geht darüber allerdings noch hinaus, denn er umfasst „a favourable attitude toward the language community, 
and an openness to other groups in general (i.e., an absence of ethnocentrism)“ (Gardner 2001: 5). In den vorliegenden Befragungen wird zielgerichtet nur der erste Teilaspekt der integrativeness geprüft, nämlich die Einstellungen zu Mehrsprachigkeit bzw. zum Spanischen im Besonderen. Dies ist deshalb ausreichend, weil im Unterschied zur Forschung über Lernmotivationen im L2Erwerb hier keine Prognose zum Lernerfolg bzw. zur Sprachfertigkeit in Abhängigkeit von der Motivation anstrebt wird, und auch keine Weiterentwicklung der didaktischen Instrumente. Im Mittelpunkt steht vielmehr eine Einschätzung des einstellungsbasierten Beitrags der Lernenden zum Language Making des Spanischen. Für diesen Beitrag sind die Einstellungen gegenüber der spezifischen Sprache und Sprachgemeinschaft entscheidend; andere Aspekte der integrativeness können demgegenüber in den Hintergrund treten. Wie genau der Anknüpfungspunkt an die Gemeinschaft aussieht bzw. wie das Wunsch zum Anknüpfen begründet wird, lässt sich grob in drei Ebenen betrachten, nämlich auf Basis materieller, instrumenteller und affektiver Zielsetzungen beim Sprachenlernen. Eine Unterscheidung von integrativeness und instrumentality, wie sie etwa Dörnyei und Csizér (2002) vornehmen, ist deshalb für den Zweck der vorliegenden Untersuchung nicht sinnvoll: Eine instrumentelle Annäherung an die Sprache kann gerade in der Zielstellung liegen, mit der Sprachgemeinschaft besser in Kontakt zu treten bzw. sich in sie einzufügen. Die drei Ebenen zur Analyse von Spracheinstellungen der Lernenden werden nach den Erläuterungen zu Einstellungen im Lernprozess weiter unten genauer beschrieben.

\subsection{Motivation und Einstellungen im Lernprozess, Sprachportfolio und Sprachennutzung}

Eine erste Kategorie der Fragebögen befasst sich mit der Lernkarriere. Gefragt wurde, ob die Teilnehmer*innen bereits vor der jetzigen Lernphase Spanisch gelernt hatten und, falls ja, in welchen Bildungsinstitutionen dies stattgefunden hatte. Sie wurden gebeten, die bisherige Dauer des Spanischlernens bzw. -studiums anzugeben sowie das Niveau des momentan belegten Sprachkurses im Gemeinsamen Europäischen Referenzrahmen (GER).

Zudem wurde erhoben, welche Kenntnisse anderer Sprachen die Befragten bereits hatten. Vorgegeben waren neben dem Deutschen einige Sprachen, die häufig oder gelegentlich an Schulen als Fremdsprachen gelernt werden (Englisch, Französisch, Russisch, Italienisch), und solche, die als Folge von Migration in Deutschland sehr präsent sind (Türkisch, Arabisch, Polnisch). Eine Kategorisierung wurde hier nicht vorgenommen, da eine trennscharfe Unterscheidung nicht möglich oder sinnvoll ist. Der Fragebogen ließ zudem genügend Raum, um weitere Sprachen zu 
nennen, die nicht vorgegeben waren. Dieses Item des Fragebogens war etwas grober gefächert als der GER, um im eingeschränkten Umfang dennoch die Möglichkeit zur Differenzierung einer größeren Zahl von Sprachen zu geben. In fünf Stufen wurde unterschieden zwischen „keine Kenntnisse“, „Grundkenntnisse (Niveau A)“, „Fortgeschritten (Niveau B)“, „Fließend (Niveau C)“ und „Muttersprache“.

Diese Auffächerung ist in vielerlei Hinsicht verallgemeinernd. Schon die Frage, wann eine Person die eigenen Sprachkenntnisse als „muttersprachlich“ auffasst, birgt großes Diskussionspotenzial (Block 2003: 80-85). Die Eigenschaft, „fließend“ eine Sprache zu sprechen, ist ebenso wie die anderen Kategorien unscharf (vgl. Burgschmidt 2005 zu Ebenen der fluency; Pomerantz 2002: 284-286 diskutiert die weit auseinandergehenden Auffassungen von proficiency beim universitären Sprachenlernen). Die Einschätzungen von Sprachkenntnissen im Rahmen der Fragebögen beruhen letztendlich auf einer Selbstwahrnehmung, oft auch im Vergleich zum sprachlichen Umfeld, zu anderen Lernenden oder zum Kontakt mit Muttersprachler`innen. Dennoch erwies sich diese vereinfachte Aufstellung für den Zweck der Befragung als nützlich, weil dadurch ein relativ klares und trotzdem differenziertes Bild der Mehrsprachigkeit der Lernenden entstand. Die Unbestimmtheit wird zudem ein Stück weit reduziert durch die Tatsache, dass den Befragten eine Skala zur Verfügung stand, so dass zumindest eine Einordnung anhand einer groben Einteilung möglich war. Für die grundlegende, größtenteils deskriptive statistische Auswertung spielten Problematiken wie beispielsweise Skalenabstände eine untergeordnete Rolle.

Mit Hilfe dieser Daten lässt sich nachweisen und berücksichtigen, dass viele Spanischlernende in der Regel bereits über Kenntnisse weiterer Sprachen verfügen. Neben dem Deutschen ist praktisch immer das Englische präsent, oft auch Französisch und in den unterschiedlichsten individuellen Kombinationen häufig noch weitere Sprachen. Mit diesen Hintergründen, die auch Spanischlernende an anderen Orten in der Welt auf ähnliche Weise mitbringen, spricht man vom Spanischen häufig nicht mehr nur als lengua extranjera oder als segunda lengua, sondern als lengua adicional (Pastor Cesteros 2016: 41). Damit lässt sich besser abbilden, dass das Spanische in ein schon breiter gefächertes Sprachportfolio einfließt, in dem es oft die dritte oder vierte erlernte Fremdsprache ist. Die umfangreiche Lernerfahrung kann nicht zuletzt wichtige Auswirkungen auf die Motivation und die Spracheinstellungen der Lernenden haben, weil sie nicht zum ersten Mal in einer Lernsituation sind und sie bereits Erfahrung mit der Herausforderung des Fremdspracherwerbs mitbringen.

Abgefragt wurde in einem gesonderten Item, ob die Lernenden sich beim Fremdspracherwerb an der europäischen oder einer lateinamerikanischen 
Form des Spanischen orientieren, oder ob sie keine Lernpräferenz im Variationsspektrum der Sprache haben. Diese Frage vereinfacht selbstverständlich stark und bildet nur einen kleinen Ausschnitt der Möglichkeiten ab, da schließlich insbesondere im lateinamerikanischen Raum eine große Vielfalt an Zielvarietäten bereitsteht (Kailuweit 2015). Diese können nach persönlichen Präferenzen und bisherigem Kontakt mit der Sprache individuell eine unterschiedliche Bedeutung für die Lernenden haben. Das Item zielt darauf $\mathrm{ab}$, zumindest ansatzweise zu erfassen, was von den Befragten als Zielnorm angesehen wird und welche Erwartung die Lernenden bei der Vermittlung einer plurizentrischen Sprache haben. Zugleich illustriert dieses Item ein breiteres theoretisches Fragefeld im Zusammenhang mit dem Begriff Plurizentrik, bei dem weiterhin recht selbstverständlich mit einer Einteilung in Natiolekte operiert wird. Mag auch die Zusammenfassung als ,lateinamerikanisches Spanisch“ sträflich grob sein, so schützt sie doch vor der ebenfalls grob rasternden Kategorisierung in ein ,argentinisches', ,peruanisches‘ oder ,kolumbianisches' Spanisch entlang nationalstaatlicher Linien. Welche Kriterien man zur systematischen Erfassung der Variation auch heranziehen mag, ist es anstelle des Nationalstaates als herausragender Variable vermutlich unverfänglicher, schlichtweg von Normvarietäten zu sprechen. Diese können wiederum durchaus gestaffelt bzw. ineinander enthalten sein, also Teil- und Untermengen voneinander bilden. ,Lateinamerikanisches Spanisch' wäre in diesem Fall ein Dachbegriff für die unterschiedlichen Normvarietäten, die in Lateinamerika gängig sind.

Die Lernenden sind unweigerlich eingebunden in den größeren Zusammenhang der Frage, ob es eine gemeinsame, weltweite Norm des Spanischen überhaupt geben kann und wie diese aussehen könnte (vgl. hierzu auch Lebsanft/ Mihatsch/Polzin-Haumann 2012). Da das Spannungsverhältnis von Variation und Norm in der Fremdsprachendidaktik kein Schwerpunkt der Untersuchung war, konnte diese Thematik im Fragebogen nicht ausführlicher behandelt werden. Wie Variation und Standardsprache(n) von Spanischlernenden wahrgenommen werden, wie sie mit Lernzielen und Spracheinstellungen zusammenhängen, bleibt deshalb ein vielversprechendes Forschungsfeld für weitergehende Untersuchungen (vgl. hierzu ausführlich bereits die Arbeiten in Leitzke-Ungerer/ Polzin-Haumann, Hg. 2017). Mit der Frage, welche spanischsprachigen Länder die Befragten bereits bereist haben, kann der Fragebogen die Bezugsräume der Lernenden andeutungsweise erfassen.

Ein bedeutender Teil des Fragebogens widmet sich der Nutzung des Spanischen. Die Befragten wurden gebeten anzugeben, wie häufig sie außerhalb des Unterrichts Spanisch sprechen und ob sie ihre Sprachkenntnisse in Deutschland nutzen oder im spanischsprachigen Ausland. Der Fragebogen gab zudem Raum für eine Aufzählung der spanischsprachigen Länder und Gebiete, welche 
die Befragten bereits besucht haben und wie lange sie sich dort aufhielten. Eine Verbindung zwischen Sprachnutzung und Lernmotivation zog die Frage nach Situationen, in denen die Befragten Spanisch sprachen oder dies zu tun planten. Vorgegeben war eine Reihe verschiedener Nutzungsbereiche wie Geschäfts- oder Urlaubsreisen, Freizeit- und Kulturangebote, Kommunikation in der Familie, im Freundeskreis oder im Beruf. Auch weitere Verwendungsbereiche konnten angegeben werden. Die Frage zielte unter anderem darauf ab, genauer einschätzen, welche Ziele die Befragten mit ihren bestehenden oder künftigen Spanischkenntnissen verfolgten, insbesondere um berufliche von privaten Motiven unterscheiden zu können bzw. die Überschneidung beider Bereiche zu erfassen.

In allen Fragebögen wurde zudem Gelegenheit gegeben, als Freitextantwort konkrete Gründe anzugeben, weshalb man Spanisch lernt. Zwar bestand hier die Möglichkeit, dass die Informationen sich teilweise deckten mit den angestrebten Nutzungsbereichen, dennoch war die Fragestellung und damit auch die gewonnenen Daten nicht völlig identisch: An dieser Stelle konnten die Befragten auch sehr individuelle Begründungen anführen, ihre Antworten nuancieren oder auch vage bleiben. Es war zudem möglich, durch einfaches Ankreuzen die Option zu wählen, dass kein konkreter Grund für das Erlernen des Spanischen vorliegt.

\subsection{Materielle, instrumentelle und affektive Komponenten von Spracheinstellungen zum Spanischen}

Ein großer Block des Fragebogens war einzelnen Elementen von Spracheinstellungen zum Spanischen als Fremdsprache und den Lernabsichten gewidmet. Den Studierenden wurden insgesamt 14 Aussagen vorgelegt, den Lernenden an den Volkshochschulen 13. Auf einer fünfstufigen Skala wurde abgefragt, ob man der Aussage zustimmt oder nicht. Die ungerade Anzahl von Zustimmungswerten wurde gewählt, um den Befragten die Möglichkeit zu geben, Unentschiedenheit und Widersprüchlichkeit auszudrücken und $\mathrm{zu}$ signalisieren, dass eine bestimmte Aussage für sie wenig zutreffend bzw. aussagekräftig ist. Der Wortlaut der einzelnen Aussagen ist in den folgenden Kapiteln bei den Datenbeschreibungen und -auswertungen jeweils wiedergegeben. Auf eine komplette Übersicht aller Aussagen der Fragebögen wird hier verzichtet, denn die Gestaltung kann je nach Zielgruppe geringfügig variieren, wenn etwa das Sprachenlernen im Rahmen eines Studiums an der Universität stattfindet oder in der Freizeit an der Volkshochschule. 
Die Aussagen decken insgesamt drei Komponenten ab, mit denen das Spanische als Ressource bewertet werden kann: eine materielle, eine instrumentelle und eine affektive. In der Forschung zu Motivationen im Fremdspracherwerb werden derartige Begründungen zum Sprachenlernen als orientations bezeichnet, um sie von der Lernmotivation als Gesamtgröße zu unterscheiden (Gardner 2001: 13-15). Dies ist im weiten Rahmen der bestehenden Forschung sinnvoll, etwa um andere Teilaspekte der integrativeness berücksichtigen zu können und um die dort vorherrschenden Erkenntnisziele zu erreichen: „There is very little evidence [. . . ] to suggest that orientations are directly associated with success in learning a second language“ (Gardner 2001: 16). Für die vorliegenden Untersuchungen, die dieses vorrangige Erkenntnisinteresse nicht teilen, ist die Unterscheidung von Motivation und Orientierung von geringerer Bedeutung und es reicht aus, hier von Einstellungskomponenten zu sprechen, die mit bestimmten Lernzielen zusammenhängen.

Die materielle Komponente entspricht am ehesten der gängigen, eingeschränkten Auffassung von Sprachen als Ressource mit einem ökonomischen Wert. Wird mit dem Erlernen des Spanischen die Erwartung oder Hoffnung auf einen wirtschaftlichen Vorteil verbunden, so bewegt sich diese Einschätzung in der materiellen Dimension der Spracheinstellungen. Hierunter fallen beispielsweise die Aussagen, man könne mit Spanischkenntnissen ein höheres Gehalt verlangen, oder man gehe davon aus, dass Spanisch für den (künftigen) Beruf erforderlich sei. Die materielle Komponente wird als Argument zum Erlernen von Fremdsprachen sehr häufig vorgebracht, von Anbietern wahrscheinlich sogar öfter als von den Lernenden selbst. Dies gilt auch für das Spanische:

[L]os distintos focos productores de discurso sobre el español parecen empeñados en instalar en la conciencia colectiva una idea fija sobre el valor económico del español y de su enseñanza como lengua extranjera.

(Bruzos Moro 2016: 2)

Mit Mehrsprachigkeit als Gut bzw. Dienstleistungskomponente können wiederum auch Unternehmen Werbung betreiben, sofern sie entsprechend qualifiziertes Personal haben:

[M]ultilingualism [. . .] itself has become a commodity given the fact that interacting in the client's language is a key marketing factor. Consequently, the recruitment of multilingual employees is becoming a clear financial advantage for companies as it is cheaper and more practical, for example, to have employees who are able to answer phone calls in more than one language.

(Duchêne 2009: 30)

In welchem Umfang die Spanischlernenden derartige Vorteile für ihre eigene (künftige) Berufstätigkeit erwarten, wird im Rahmen der Befragungen geprüft. 
Auch in der weiter unten beschriebenen Befragung von Beschäftigten im Berliner Gastgewerbe wird diese Frage eine zentrale Rolle spielen.

Die instrumentelle Komponente der Bewertung umfasst die Einschätzung, dass mit Hilfe der spanischen Sprache die Kommunikation verbessert oder erleichtert werden kann. ${ }^{28}$ Hierunter fallen also auch Einschätzungen, die keinen unmittelbaren ökonomischen Vorteil verfolgen. ${ }^{29}$ Beispiele hierfür sind das Ziel, spanischsprachige Literatur im Original lesen zu können oder die Erwartung, mit Hilfe des Spanischen neue private Kontakte zu knüpfen. In diese Kategorie passt auch die Frage, ob die Lernenden sich eher auf die schriftliche oder mündliche Kommunikation konzentrieren, oder beide Dimensionen gleich gewichten. Damit lässt sich einschätzen, auf welche Art sie das Spanische als Kommunikationsinstrument nutzen möchten, beispielsweise welche Rolle der Bereich des Schriftstandards spielt. Ebenfalls an die instrumentelle Komponente angebunden ist die Aussage, Spanischkenntnisse seien nicht notwendig, wenn man gute Englischkenntnisse hat. Daran lässt sich der Wert des Spanischen als Kommunikationsinstrument in den Augen der Befragten im Verhältnis zum Englischen ablesen.

Die instrumentelle und die materielle Dimension sind nicht unbedingt klar voneinander trennbar. ${ }^{30}$ Ein Item im Fragebogen bestand etwa aus der Aussage,

28 De Swaan (2010: 58-61) stellt mit dem sogenannten Q-value eine Formel auf, mit der sich die kommunikative Reichweite einer Sprache einschätzen lassen soll. Ausgangspunkt ist in erster Linie die Anzahl mehrsprachiger Sprecher`innen, in deren Sprachrepertoire die zu beschreibende Sprache vorkommt. Der Wert beschreibt dann für eine Sprachkonstellation und einen gegebenen Sprecher das „potential to link this speaker with other speakers in $S$ [der gegebenen Sprachkonstellation, P.K.]“ (De Swaan 2010: 58). Ob ein solcher Q-Wert für die befragten Spanischlernenden aussagekräftig wäre, muss an dieser Stelle offen bleiben. Nicht einbezogen sind in dem Wert jedenfalls wichtige Einflussfaktoren wie etwa die Frage, inwiefern verschiedene Kompetenzniveaus der Sprecher`innen einen solchen Wert beeinflussen oder in welchem Ausmaß Spracheinstellungen die Bereitschaft zur Verwendung bestimmter Sprachen vergrößern oder verringern.

29 In der Forschung zu Lernmotivationen wird dies bisweilen anders gewichtet bzw. der ökonomische Nutzen als Hauptmerkmal für instrumentelle Herangehensweisen des Sprachenlernens gesehen, vgl. beispielsweise Dörnyei/Csizér (2002).

30 Die Übergangsbereiche treten noch stärker zutage, wenn man wie Gardner (2001: 14) nur von zwei Ebenen ausgeht, nämlich einer instrumentellen und einer integrativen. Die instrumentelle Ebene entspricht dabei vor allem demjenigen, was hier als materielle Ebene beschrieben ist. Der Wunsch zur besseren Kommunikation kann dann aber nur entweder unter materiellen Gesichtspunkten betrachtet werden und wird dann instrumentell genannt, oder aber er ist von solchen Zielstellungen befreit und gilt demnach als integrativ. Eine Überschneidung zwischen beiden ist so schwer abzubilden (vgl. hierzu auch Noels 2001: 44). Deshalb wurde für die vorliegenden Befragungen ein Modell mit drei Ebenen gewählt, bei dem Übergänge mitgedacht werden können. 
mithilfe des Spanischen wolle man neue berufliche Kontakte gewinnen. Dies kann auf einen direkten materiellen Vorteil abzielen, möglicherweise ist die Wertung des Spanischen als instrumentelle Ressource aber deutlich allgemeiner und es geht nur darum, mit einer zusätzlichen Kommunikationsmöglichkeit das persönliche Netzwerk auszubauen. Vogl (2018a, b) unterschied bei Studierenden im Sprachenlernen zwischen den Dimensionen qualification und functionality. Die erste Dimension liegt dabei genau an der Schnittstelle zwischen der materiellen und der instrumentellen Komponente, soweit damit Sprachkenntnisse zur Nutzung im Beruf gemeint sind; functionality umfasst die Anteile der instrumentellen Komponente, welche insgesamt die Kommunikationsfähigkeit betreffen und nicht nur auf das Berufsleben begrenzt sind.

Als dritte Komponente kann die Bewertung des Spanischen auf affektiver Basis vorgenommen werden. Die Sprache ist dann in einem relativ weiten Sinne eine Ressource, deren Wert darin liegt, dem oder der Sprecher*in positive Emotionen $\mathrm{zu}$ bereiten. ${ }^{31}$ Inwiefern eine emotive Bindung an die Sprache besteht, prüft beispielsweise die Aussage, dass die Befragten anstelle des Spanischen auch eine andere Sprache hätten wählen können oder dass sie bereits seit längerer Zeit den Beschluss gefasst hatten, Spanisch zu lernen. Die affektive Bewertung der Sprache wird im Fragebogen zudem auf Basis von Ästhetik erfasst. Im Zentrum steht die Aussage, Grund für das Erlernen des Spanischen sei die Schönheit der Sprache. Von Schüler^innen der 9. und 10. Klasse an Realschulen wurde das Spanische als besonders sympathische Sprache gewertet, noch vor dem Englischen (Plewnia/Rothe 2011: 226). Die Befragten der Untersuchung von Plewnia und Rothe sind heute ungefähr im jungen Erwachsenenalter, also etwa in der gleichen Generation wie jetzige Studierende. Zwar deckt sich die Formulierung der Fragen nicht exakt mit jenen der Studie von 2011, dennoch kann erkundet werden, ob die sehr positiven Zuschreibungen auch bei heutigen Studierenden und bei Lernenden an Volkshochschulen vorzufinden sind. Die Fragen im affektiven Bereich und einige Items aus dem Bereich zum Vergleich zwischen Spanisch und Französisch (s. weiter unten) lehnen sich damit lose an das „Allgemeine Sprachbewertungsinstrument“ (ASBI) an, das in Adler/Plewnia (2018: 69-79) erläutert wird.

Auch bei der affektiven Dimension besteht ein Übergangsbereich, vor allem zur Bewertung des Spanischen als instrumentelle Ressource. Die Nutzung der

31 In anderen Forschungszusammenhängen werden derartige Lernmotivationen häufig als ,intrinsic“ bezeichnet (vgl. beispielsweise Noels 2003). Um das Missverständnis zu vermeiden, dass die Motivation als der Sprache und nicht den Sprecher*innen intrinsisch angesehen wird - also dass die Motivation aus inhärenten Qualitäten der Sprache ableitbar wäre -, soll hier an dem weniger fest eingeführten Begriff affektiv festgehalten werden. 
Sprache zur Lektüre von Literatur in Originalsprache kann beispielsweise aus dem Wunsch eines besonderen ästhetischen Erlebnisses in der Fremdsprache erwachsen. Eine affektive Komponente hat auch der Wunsch, die Möglichkeiten zur Kommunikation mit Nahestehenden wie im Freundes- und Familienkreis zu erweitern, wenn mit der Sprache Nähe, Freundschaft oder Vertrautheit verbunden bzw. hergestellt werden soll.

In der Spracheinstellungsforschung werden Einstellungen $\mathrm{zu}$ bestimmten Sprachformen oder Sprecher*innen klassischerweise in die beiden Dimensionen status und solidarity eingeteilt:

The first, termed status (or competence), encompasses traits such as a speaker's intelligence, education, and success. The second, termed solidarity (or warmth), encompasses traits such as a speakers' friendliness, pleasantness, and honesty. Different language varieties are associated with different stereotypes along these dimensions.

(Dragojevic 2017: 9)

Die drei Dimensionen, welche die folgenden Untersuchungen leiten, lassen sich zu einem gewissen Teil daran anschließen: Sofern mit dem Erlernen des Spanischen materielle Ziele verfolgt werden, wird die Sprache aus einer statusbetonten Perspektive beurteilt. Die affektiven Bewertungen schließen vor allem an die Ebene der Solidarität an, wenn das positive persönliche Erlebnis beim Kontakt mit der Sprache bzw. der Sprachgemeinschaft im Mittelpunkt steht. Die instrumentelle Dimension als Übergangsbereich kann je nach Fragestellung näher an einem der beiden Pole stehen. Wird das Spanische eher als Instrument etwa für beruflichen Aufstieg gesehen, steht die instrumentelle Bewertung der StatusKomponente näher; sofern es als Instrument zur interkulturellen Kommunikation etwa im privaten Bereich betrachtet wird, wiegt die Seite der Solidarität schwerer. Im letzteren Fall spielt vor allem eine Rolle, dass die Lernenden danach streben, mithilfe des Spanischen ein gewisses Maß an in-group-Bindung mit der spanischen Sprachgemeinschaft zu erreichen, wenn sie an deren Sprache teilhaben.

Ähnlich lässt sich auch eine Zuordnung zu einer weiteren etablierten Dichotomie vornehmen, die in der Forschung zur Motivation beim Fremdsprachenlernen gebraucht wird. Noels (2001: 45-46, 51) unterscheidet als Gründe zum Fremdsprachenlernen zwischen intrinsic und extrinsic orientations. Erstere zielen auf die Erlangung von persönlicher Zufriedenheit und auf die Befriedigung individueller Interessen ab; zweitere decken hingegen Lerngründe $a b$, die von außerhalb an die Lernenden herangetragen werden - im Extremfall beispielsweise die reine Verpflichtung zum Sprachenlernen durch bestehende Regelungen zum Schulunterricht oder erforderliche Berufsqualifikationen. Innerhalb dieser Unterscheidung liegen letztendlich alle hier geprüften Einstellungselemente auf der Seite der intrinsischen Orientierung, weil es für die Befragten in Berlin keine verbindliche 
Regelung zum Erlernen des Spanischen gibt. Dennoch können auch extrinsische Gründe internalisiert werden (Noels 2001: 46). Hierzu zählt beispielsweise die starke Wirkmacht des Diskurses von Sprachen als ökonomisch verwertbare Qualifikation. Auch wenn selbst materiell orientierte Spracheinstellungen auf einen individuellen Vorteil abzielen, werden sie doch stark von außen gespeist. Tendenziell kann man daher in einem Kontinuum von materiellen, instrumentellen und affektiven Annäherungen davon ausgehen, dass die affektiven Komponenten am stärksten auf der Seite der intrinsischen Gründe liegen, während die extrinsischen Kräfte in Richtung der materiellen Einstellungen ein wenig zunehmen. Eine eindeutige Trennung der drei Komponenten mit einer Zuordnung zur Unterscheidung zwischen intrinsischen und extrinsischen Gründen lässt sich allerdings nicht vornehmen; dies ist auch in den bestehenden Forschungsrahmen zur L2-Lernmotivation nicht vorgesehen.

Eine letzte Spracheinstellungsfrage, die unmittelbar mit dem Erwerbsprozess als solchem zusammenhängt und nicht an die drei oben genannten Dimensionen anschließt, wurde in den Fragebögen für die Studierenden an der Freien Universität und der Technischen Universität noch angefügt; in den Fragebögen der Lernenden an Volkshochschulen war dieses Item noch nicht vorhanden. Vorgegeben war die Aussage „Ich lege Wert darauf, dass mein/e Kursleiter/in im Spracherwerbskurs muttersprachliche Spanischkenntnisse hat.“ Von Lernenden und Lehrenden zugleich wird die Deutungshoheit und Legitimität bei der Sprachvermittlung häufig vorrangig denjenigen zugeschrieben, die in der Sprachgemeinschaft bereits seit früher Kindheit fest verwurzelt sind. Diese Ansicht beruht auf der Annahme, „dass Muttersprachler_innen eine überlegene Sprachkompetenz gegenüber Nicht-Muttersprachler_innen haben, dass muttersprachliche Kompetenz das Lernziel im Sprachunterricht sein sollte und dass Muttersprachler_innen die geeignetsten Sprachlehrer_innen sind“ (Becker 2018: 194). Vor diesem Hintergrund war es interessant zu erfahren, ob die Spanischlernenden der Berliner Universitäten diese Denkweise teilen. Holliday (2006) zeigt für das Englische, dass mit solchen Haltungen des „native speakerism“ auch tiefergehende Überzeugungen verbunden sein können, z. B. die Ansicht, dass das Englische in erster Linie von Sprecher^innen mit einem ,westlichen“ Kulturhintergrund vertreten werde. Für das Spanische ergibt sich daraus eine interessante Frage vor allem im Zusammenhang mit den Lernpräferenzen im Hinblick auf europäische oder lateinamerikanische Normen: Hier besteht eine Möglichkeit, dass Sprecher^innen aus dem globalen Süden deutlich eher als Lehrautoritäten aufgefasst und anerkannt werden, als das beim Englischen der Fall ist. Das einzelne Item im Fragebogen kann diese Problematik selbstverständlich nicht umfassend aufarbeiten, man kann daran aber weiteren Forschungsbedarf ausmachen. 


\subsection{Spanisch und Französisch im Vergleich}

Ein weiterer Block der Fragebögen war speziell dem Verhältnis von Spanisch und Französisch gewidmet. Der Block bestand aus insgesamt zehn Kategorien, für die entschieden werden sollte, ob sie eher auf das Französische, auf das Spanische oder auf beide Sprachen gleichermaßen bzw. keine von beiden zutraf. Eingeleitet wurde der Block mit der folgenden Erläuterung:

Französisch und Spanisch sind beides beliebte Fremdsprachen. Wir möchten gern erfahren, wie Sie die beiden Sprachen im Vergleich wahrnehmen. Bitte kreuzen Sie an, auf welche Sprache nach Ihrem persönlichen Eindruck die folgenden Punkte eher zutreffen.

Der Vergleich zwischen Französisch und Spanisch zielt vor allem auf Statuszuweisungen an die beiden Sprachen ab. Geprüft werden diese anhand von Labels bzw. Schlagworten, die zum Teil auf etablierte Begriffe zurückgreifen, zum Teil auch weniger geläufige Konzepte abbilden.

$\mathrm{Zu}$ den am weitesten verbreiteten Labels zählt sicherlich der Begriff Weltsprache, der im Zusammenhang mit dem Konzept des Global Spanish eine wichtige Rolle spielt, wenn der weltumspannende Charakter der Sprache mit ihren vielen verschiedenen Funktionen und Ausprägungen sowie Bewertungen im Rahmen von Spracheinstellungen untersucht werden soll.

Im Rahmen der Diskussion um weltweite Kunstsprachen etwa zur Wende zwischen 19. und 20. Jahrhundert widmete sich der Romanist Hugo Schuchardt im Dialog mit dem Indogermanisten Gustav Meyer der Frage, was Weltsprachen seien. Schuchardt kommt zu einer Minimaldefinition, die er in Abgrenzung von der damals aktuellen Debatte um das Volapük formuliert: „[. . .] wir Uebrigen verstehen unter ,Weltsprache“ oder wie ich wegen der Doppeldeutigkeit dieses Wortes vorziehe zu sagen ,Allgemeinsprache‘ eine internazionale Sprache neben den nazionalen Sprachen" (Schuchardt 1894: 8). Zu den internationalen Sprachen zählt Schuchardt auch Spanisch, Französisch oder Englisch, die zur damaligen Zeit schon weltumspannende Verbreitung besaßen. Die Bindung einer Sprache an eine Nation entspricht der prägenden Ansicht im 19. Jahrhundert, die bis heute wirksam bleibt. Bei der Lösung dieser Verbindung sollten künstliche Sprachen helfen. In der Tat zeigt sich auch heute noch, dass bei den natürlichen Sprachen, die gegenwärtig als Weltsprachen gesehen werden, trotz ihrer internationalen Verwendung eine gewisse Vorstellung von ,Herkunftsnationen“ oder ,Mutterländern' bestehen bleibt.

Bei der Feststellung der Reichweite und Anzahl von Sprecher^innen sogenannter ,großer Sprachen ist spürbar, dass ,a menudo se presenta el español de modo triunfalista“ (Paster Cesteros 2016: 42). Das Label Weltsprache lässt sich also nicht komplett vom Gedanken der Hegemonie lösen. Die meisten Sprachen, 
die heute als Weltsprachen gelten, - zumindest diejenigen europäischer Herkunft - haben diesen Status durch koloniale Expansion erhalten. ${ }^{32}$ Dies gilt jedenfalls für das Spanische wie für das Französische gleichermaßen. Zugleich lässt sich außerhalb Europas beobachten, wie die ehemaligen Kolonialsprachen appropriiert und transformiert werden, was beispielsweise den plurizentrischen Charakter der Sprachen stärken kann (Lebsanft 1996: 213). Ob von Spanischlernenden einer der beiden Sprachen trotz ihrer relativ vergleichbaren weltweiten und historischen Stellung eine unterschiedliche Bedeutung zugeschrieben wird, kann mit der vergleichenden Abfrage im Fragebogen geprüft werden.

Man kann im Prinzip davon ausgehen, dass Weltsprachen - so man dies als gültige Kategorie ansieht - mehr oder weniger automatisch dazu tendieren, zu plurizentrischen Sprachen zu werden. Ihre Verbreitung und frequente Nutzung führt praktisch unweigerlich dazu, dass sich Normunterschiede herausbilden. Dass die Forschung zu plurizentrischen Sprachen lange Zeit am Nationalstaat als erklärende Größe für die Einteilung verschiedener Normvarietäten festhielt, hat die Erklärungskraft des Konzepts bisweilen eingeschränkt (Corti 2019: 91-92). Zugleich liefert der konsequente Übergang hin zu einer pluriarealen Betrachtung von Variation in Sprachnormen nicht immer befriedigende Alternativen, wenn damit die politischen oder ökonomischen Grundstrukturen zu sehr in den Hintergrund geraten (Dollinger 2019). In der vorliegenden Studie zu den Einstellungen von Spanischlernenden ist der Bereich von Variation und Standard nur ein kleinerer Aspekt, so dass Debatten über das Konzept Plurizentrik hier keinen größeren Raum einnehmen müssen. Es bietet sich jedoch an, diesen Bereich in der Fremdsprachenforschung des Spanischen künftig auszubauen.

Der Begriff Weltsprache transportiert eine große Bandbreite von Zuschreibungen, welche im weitesten Sinne die ,Bedeutung der Sprache zu erfassen versuchen (Ammon 2010, Schnitzer 2012: 157). Sie sind geknüpft an die Größe der Sprachgemeinschaft, die geographische Verbreitung, die ökonomischen Potenziale und insgesamt an die kommunikative Reichweite der Sprache insbesondere in der internationalen und sprachgruppenübergreifenden Kommunikation. ${ }^{33}$

32 Es zeigt sich hier, dass nicht ohne Weiteres gültig ist, was J. Leonhardt (2009: 15) mit Blick auf das Lateinische als Weltsprache feststellt: „Die bloß gewaltsam durchgesetzte Verbreitung einer Sprache macht noch keine Weltsprache; wenn die Weltmacht zusammenbricht, ist auch die Sprache am Ende.“ Dass es keinen Automatismus gibt, liegt auf der Hand - allerdings zeigt das Beispiel des Spanischen ebenso wie des Portugiesischen oder Französischen die Langlebigkeit der Sprachverwendung und sprachlichen Dominanz auch über das Ende der kolonialen Ära hinaus.

33 De Swaan (2010: 56-57) spricht von „central languages“, wenn diese häufig als Zweitsprache von Sprecher^innen aus kleineren Sprachgemeinschaften erworben werden und dann zur gruppenübergreifenden Kommunikation dienen. Das Spanische klassifiziert er gemeinsam mit etwa einem Dutzend anderen Sprachen aufgrund der besonders großen Reichweite über viele 
Die Reichweite solcher Sprachen kann im Vergleich untereinander relativ sein und sie erstreckt sich in der Regel gerade nicht auf die gesamte Welt, sondern auf größere Weltregionen (Sinner 2013: 5-7). Genau dies wiederum bringt mit sich, dass es neben dem Englischen weiterhin mehrere Weltsprachen geben kann und dass ein Vergleich zwischen Spanisch und Französisch überhaupt möglich ist (vgl. Ammon 2010, der das Nebeneinander von Weltsprachen im Plural betont). Für das Spanische umfasst das Gebiet seiner ,klassischen“ Reichweite unter anderem praktisch den gesamten amerikanischen Kontinent von Nord bis Süd, weil Spanisch als wichtige Fremd- und/oder Erstsprache auch in den nicht-hispanophonen Ländern der Karibik, in den USA und Kanada oder in Brasilien verbreitet ist (Schnitzer 2012: 159). Das Beispiel USA zeigt dabei, dass L1- und L2-Sprachgebiete keineswegs klar voneinander trennbar sind. Die weltweit sichtbare Beliebtheit des Spanischen als Fremdsprache deutet zudem darauf hin, dass die Annahme eines abgeschlossenen Sprach- oder auch Einflussgebiets gerade für Weltsprachen ohnehin problematisch ist.

In der nicht-akademischen Verwendung verschmilzt der Begriff Weltsprache häufig mit Konzepten wie der einer (internationalen) Verkehrssprache (vgl. umfassend hierzu, insbesondere mit Blick auf das Spanische, Sinner 2013; Lebsanft 1996). Mit ähnlichen Kenngrößen charakterisiert Marcos-Marín (2006: 32-33) das Spanische auch als lengua internacional. Die Bewertung der entscheidenden Kriterien dürfte subjektiv und individuell variieren, je nach persönlicher Wahrnehmung und Erfahrung mit verschiedenen Sprachen in unterschiedlichen Gebieten der Welt, so dass die Einstufung des Spanischen und Französischen als ,Weltsprachen' im Sinne einer einstellungsbasierten Zuschreibung prüfbar ist.

In Abstufungen etwas weniger geläufig bzw. überhaupt nicht fest etabliert sind die ebenfalls abgefragten Begriffe Kultursprache und Wirtschaftssprache. Die damit verbundenen Konzepte fließen in der Regel in die Bewertung einer Sprache als Weltsprache mit ein, sie greifen dabei aber bestimmte Bereiche noch einmal gezielt heraus. Der Begriff Wirtschaftssprache ist mehr als die beiden anderen ein spontan verkürztes Label. Man kann man davon ausgehen, dass die Befragten intuitiv ein Konzept mit dem Begriff verbinden konnten, indem sie ihre Wahrnehmung der wirtschaftlichen Bedeutung der Sprache, in Berufsleben, im weltweiten Handel und im global verflochtenen Wirtschaftssystem einschätzen. Im Hinblick auf dieses Kriterium ist das Spanische aufgrund der bisweilen sozioökonomisch relativ schwachen Stellung etwa einiger lateinamerikanischer Staa-

Sprachgemeinschaften hinweg als „supercentral language“. Da der Begriff gerade im Zusammenhang mit dem Begriff der Plurizentrik verwirrend sein kann, wird er hier nicht weiter verwendet. 
ten deutlich schwieriger einzuordnen als wenn nur die demographische Größe der Sprachgemeinschaft herangezogen wird (Sinner 2013: 17-18).

In die Bewertung einer Sprache als Kultursprache fließt der Umfang der kulturellen Produktion ein, etwa von Literatur bzw. Verlagswesen, Übersetzungen, Film und Fernsehen, Musik und Medien in der jeweiligen Sprache (Sinner 2013: 19-20, Schnitzer 2012: 162). Nicht selten gilt dabei eine kleine Anzahl kanonischer Schriftsteller (und seltener Schriftstellerinnen) als emblematisch, wenn nicht gar konstitutiv, für die Qualität einer Sprache als Kultursprache. Häufig werden so Goethe und Schiller mit dem Deutschen in Verbindung gebracht, Molière oder Voltaire mit dem Französischen, Shakespeare mit dem Englischen und Cervantes mit dem Spanischen (vgl. Lebsanft 1996: 211).

Bei beiden Konzepten, Kultur- und Wirtschaftssprache, darf wiederum die subjektive Komponente nicht unterschätzt werden, hängt die Bewertung einer Sprache doch stark von der Wahrnehmung der einzelnen Person ab, etwa von der Intensität, mit der man sich mit den entsprechenden Kulturformen auseinandersetzt oder am Wirtschaftsleben im Sprachgebiet teilnimmt.

Wichtig ist bei diesen Kategorien ebenso wie bei den unten weiter erläuterten Zuschreibungen: Es ist nicht das Ziel, zu einer wissenschaftlich objektiven Messung der Bedeutung des Spanischen oder des Französischen zu kommen, mit der den beiden Sprachen ein absoluter Wert zugewiesen werden könnte. Lebsanft (1996) hat solche Versuche und Tendenzen in der Wissenschaft zu Recht kritisiert, insbesondere mit Blick auf den Anspruch, dem Spanischen als Kultur- und Weltsprache eine besondere Förderung und Pflege zukommen zu lassen. Vielmehr soll erfasst werden, ob und wie solche Zuschreibungen außerhalb der Forschung auf die beiden Sprachen angewandt werden, denn für Lernende, Lehrende und für die Sprachgemeinschaften können die damit verbundenen Haltungen äußerst wirksam sein. Dies schließt selbstverständlich nicht aus, dass Antworten im Rahmen der Befragung auf Basis eines wissenschaftlich informierten Urteils zustande kamen.

Als weitere Zuschreibungen wurden für das Französische und Spanische im Fragebogen die Attribute als ,schwierige, schöne, wichtige, erotische, fröhliche, komplizierte“ Sprache sowie als „Fremdsprache, die in Deutschland jeder lernen sollte“ angeboten. Die Eigenschaften zielten vorwiegend auf die affektiven und instrumentellen Einstellungsdimensionen sowie auf Einschätzungen des Lernprozesses ab. Französisch gilt bei Deutschsprachigen häufig als schwer zu erlernende Sprache (Sigott 1993: 24, Polzin-Haumann 2015, Venus 2017: 133, Schwender 2018). Inwiefern dies eine diskursiv hervortretende Einstellungsfrage ist, soll mit den Zuschreibungen als ,komplizierte“ und „schwierige“ Sprache getestet werden. Da Spanisch und Französisch sich strukturell stark ähneln, ist es 
besonders interessant $\mathrm{zu}$ betrachten, ob beide Sprachen auch als ähnlich schwer oder leicht zu erlernen angesehen werden.

Mithilfe der Aussage, das Französische, das Spanische oder beiden Sprachen sollten in Deutschland von allen gelernt werden, lässt sich genauer erfassen, ob die Befragten diese Sprachen in erster Linie als Ressourcen für sich selbst ansehen, die also an das Individuum gebunden sind, oder ob sie den Status als wertvolle Ressource als objektiv bzw. übertragbar betrachten.

In Ansätzen ähneln einige der Zuschreibungen der Vorgehensweise der Attitudes-towards-Languages-Skala (AToL), genauer gesagt den damit gemessenen Dimensionen value, welche $\mathrm{u}$. a. affektiv-ästhetische Bewertungen einschließt, und structure, bei der die Wahrnehmung strukturlinguistischer Eigenschaften gemessen wird (Schoel et al. 2013, Plewnia/Adler 2018: 79-89). Im Unterschied zu den AToL-Instrumenten wurden hier keine skalaren Einordnungen vorgegebener Attribute für beide Sprachen abgefragt, sondern lediglich ein absoluter Vergleich zwischen den beiden beteiligten Fragen, um den Umfang und Aufwand für die Befragten in einem vertretbaren Rahmen zu halten. Ein genauerer Vergleich zwischen Französisch und Spanisch im Rahmen der AToL-Methodik bleibt daher noch ein Forschungsdesiderat.

\subsection{Methodische Grundfragen: Datenerhebungen und Auswertung}

Die oben beschrieben Gliederung der Fragebögen soll zumindest näherungsweise der Tatsache Rechnung tragen, dass (Sprach-)Einstellungen ein komplexes Geflecht von Wertungen bestimmter Einzelaspekte bilden, die zusammenwirken oder einander widersprechen: „Perhaps a person has many stored associations with a particular attitude object, and these stored associations each have evaluative implications. But, for whatever reason, these evaluative implications have never been integrated or crystallized into a single evaluative summary stored in memory.“ (Krosnick/Judd/Wittenbrink 2018: 47). Es ist also im Prinzip unmöglich, die Einstellung schlechthin einer Person zum Spanischen zu erfassen. Stattdessen kann man lediglich antizipieren, welche Elemente von Spracheinstellungen eine Rolle spielen können und diese in einem möglichst kohärenten Gesamtbild zusammentragen.

Zur Erhebung von Daten zu Spracheinstellungen und Motivationen der Spanischlernenden wurde eine explizite Methode gewählt. Die Befragten wurden gebeten, auf Fragebögen unmittelbar ihre Einschätzungen zu verschiedenen Aspekten des Sprachenlernens, der Wahrnehmung von Sprache(n) und der sprachlichen Praxis zu äußern. Eine implizite Methode war in diesem Rahmen 
nicht nötig bzw. überhaupt nicht durchführbar, weil die Teilnehmenden selbst einbegriffen waren in der Sprachgemeinschaft, zu der sie befragt wurden. ${ }^{34} \mathrm{Da}$ die Forschungsfrage auf die Beteiligung der Lernenden am Language Making des Spanischen abzielt und diese daher auch ihre eigene Rolle als Sprecher ${ }^{\star}$ innen mit einbezogen, hätte eine implizite Methode etwa mit einer Projektion auf eine Sprachgemeinschaft als rein ,externe‘ Größe zu verfälschten Eindrücken geführt. Selbstverständlich bedeutet auch eine explizite Herangehensweise immer, dass Erkenntnisse zu Spracheinstellungen ausschließlich durch Interpretation zugänglich sind: „Because attitudes, like all psychological constructs, are latent, we cannot observe them directly. So all attitude measurement depends upon those attitudes being revealed in overt responses, either verbal or nonverbal.“ (Krosnick/Judd/Wittenbrink 2018: 46)

Die Fragebögen wurden in Papierform verteilt und in den jeweiligen Lerngruppen zum gleichen Zeitpunkt ausgefüllt. An den Universitäten wurden die Fragebögen von den Lehrenden an die Lerngruppen ausgegeben; an den Volkshochschulen teils von den Dozent*innen und teils von einer studentischen Mitarbeiterin der Freien Universität Berlin, die hierzu mit Zustimmung der Lehrenden und der Volkshochschulen den Unterricht besuchte. Tab. 6.1 gibt einen Überblick über den Zeitpunkt der Erhebung in den drei Gruppen sowie die Anzahl der vollständigen Datensätze.

Tab. 6.1: Erhebungen zu Spracheinstellungen von Spanischlernenden in Berlin.

\begin{tabular}{llr}
\hline Lernort / Zielgruppe & Erhebungszeitraum & $\begin{array}{r}\text { Anzahl } \\
\text { Befragte }\end{array}$ \\
\hline $\begin{array}{l}\text { Studierende im Fach Spanische Philologie } \\
\text { bzw. Spanisch mit Lehramtsoption an der } \\
\text { Freien Universität Berlin }\end{array}$ & Sommersemester 2018 & 31 \\
\hline $\begin{array}{l}\text { Studierende in Spanischkursen der } \\
\text { Sprach- und Kulturbörse an der }\end{array}$ & $\begin{array}{l}\text { Frühjahr/Sommer 2017 } \\
\text { (Kurse im Sommersemester 2017, }\end{array}$ & 54 \\
Technischen Universität Berlin & $\begin{array}{l}\text { Intensivkurse in den vorausgehenden } \\
\text { Semesterferien) }\end{array}$ & \\
\hline Teilnehmer*innen an Spanischkursen von & Kurse mit Laufzeit von Herbst 2016 bis & 102 \\
Volkshochschulen in Berlin & Frühjahr 2017 & \\
\hline
\end{tabular}

34 Vgl. Meyerhoff/Schleef/MacKenzie (2015: 83-84) zu Potenzialen und Grenzen direkter wie indirekter Methoden in der Spracheinstellungsforschung; zu expliziten und impliziten Methoden in der psychologischen und sozialwissenschaftlichen Einstellungsforschung vgl. ausführlich auch Krosnick/Judd/Wittenbrink (2018). 
Die Aussagekraft der Stichproben hängt insbesondere davon ab, welche Personenkreise man als Grundgesamtheit annimmt. So stellen die Befragten an der Freien Universität Berlin einen sehr großen Anteil des gesamten Jahrgangs im Spanischstudium an dieser Universität; schon im Vergleich zur benachbarten Humboldt-Universität können die Spracheinstellungen und die persönlichen Hintergründe sich erheblich unterscheiden, weil beide Universitäten potenziell verschiedene Zielgruppen als Studierende ansprechen und anziehen. Zumindest kann jedoch gesagt werden, dass die Befragten in den drei Stichproben jeweils in zentralen Merkmalen mit ihrer jeweiligen Gesamtgruppe übereinstimmen, beispielsweise was die Altersstruktur und Zusammensetzung nach Geschlechtern betrifft, im Falle der TU Berlin die Fachhintergründe der Studierenden (großer Anteil im technischen und mathematisch-naturwissenschaftlichen Bereich), oder im Falle der Lernenden an Volkshochschulen deren Bildungshintergrund (großer Anteil von Kursteilnehmer*innen mit hohem formalem Bildungsgrad). Repräsentativität für größere Grundgesamtheiten, etwa für alle Sprachenlernenden in Berlin oder gar die Gesamtbevölkerung Berlins, kann selbstverständlich nicht angenommen werden (Adler/Plewnia 2018: 64).

Die Daten wurden im Anschluss an die Erhebung digitalisiert und mit Hilfe von R Studio ausgewertet. Zur graphischen Bearbeitung wurden einige der Ergebnisse rückübertragen in Microsoft-Anwendungen.

Im Mittelpunkt stand bei der Auswertung vor allem ein grundlegendes Niveau deskriptiver Statistik. Zur Erfassung von zentralen Informationen wie Lernhintergründen, -zielen und -fortschritten genügt in der Regel bereits eine Betrachtung der Verteilung, die im Folgenden in der Regel als absoluter Zahlenwert wiedergegeben wird. Visualisierungen und beigefügte Interpretationen machen häufig die Anwendung tiefgreifender Verfahren etwa der analytischen Statistik überflüssig bzw. durch geringe Fallzahlen kleinerer Teilgruppen innerhalb der Gesamtheit der Befragten schwer zu operationalisieren. Lediglich im Bereich von Spracheinstellungen oder bestimmten zielgruppenspezifischen Einzelfragen erweist sich die Überprüfung beispielsweise von Korrelationen hin und wieder als sinnvoll und aussagekräftig.

Mit einzelnen Freitextfragen, in die meist nur wenige Schlagwörter einzutragen waren, enthielten die Fragebögen auch einen geringfügigen qualitativen Anteil. Diese konnten durch Kategorisierungen häufig wieder in eine quantitative Auswertung rücküberführt werden. Dies gilt beispielsweise bei der Aufzählung spanischsprachiger Reiseziele, bei der Nennung von Lernzielen oder von erwarteten Verwendungsbereichen der Sprache. 